\title{
Slide Development of Remote Control Camera with Bluetooth Communication
}

\author{
Joon-Ho Cho
}

\begin{abstract}
In this paper, we developed a slide imaging system that compensates for tremor when shooting using a camera. It uses a microprocessor to control the stepping motor, and can control slideshows with smartphone apps. We also developed an object tracking method, which is an image processing technique, to automatically capture human motion. It is not easy to obtain a clear image by camera shake when photographing an object with a camera. Some products have recently added camera shake correction function, but products added with such functions are often expensive in price. For these reasons, video equipment sliders are also used by video shooting experts and the general public. However, the conventional products have troublesomeness that people have to move directly. Electric sliders created to solve such inconveniences need to be attached to rails and controlled with buttons, if a motion controller is added. In this thesis, we developed the electric slider that operates with the button using Bluetooth telecommunication of the smartphone so that the position and speed of the slider can be controlled, and in order to reduce the blur, the stepping motor Control.
\end{abstract}

Keywords : Bluetooth, ,PID controller, Camera slider, Stepping motor, Telecommunication

\section{INTRODUCTION}

Theworld's first electronic camera, since Mavica Sony announced in 1980, the camera's image sensor has been continuously reduced in size and high in image quality. However, as a result of high performance, miniaturization and weight saving of digital cameras, cameras have been very sensitive to vibration (camera shake) applied to the camera from the outside. In the case of a compact or high-end type digital camera, camera shake phenomenon caused by a structural problem causes deterioration of the image quality, and even in a very skilled photograph, in all situations the phenomenon that the camera shakes in camera shake Cannot be prevented fundamentally. So when taking pictures, users are sensitive to shutter speed at any time. There are various methods for camera shake correction technology, but it can be largely classified by optical correction method and mechanical correction method [1-4].

In the method of adjusting the optical path by moving an additional correction lens in accordance with camera shake, the optical correction method is also called lens detachment because it corrects blur by moving the lens. Although this method can effectively reduce image distortion, this method

Revised Manuscript Received on July 22, 2019.

* Correspondence Author

Joon-Ho Cho*, Department of Electronics Convergence Engineering, Wonkwang University, Iksan, Republic of Korea, Email cho1024@wku.ac.kr requires extra lenses, so it is mainly mounted on the lens itself, not the body of the camera. A mechanical correction method is also called a movement method of an image sensor in a method in which light incident from a subject moves to the center of the image sensor by moving the image sensor so as to detect camera shake and compensate for it It is. Unlike the optical correction method, the mechanical correction method can not maintain the orthogonality between the optical axis and the image sensor. However, since it is advantageous for size reduction and weight reduction, it is mainly applied to small digital cameras. The camera shake correction technology is a well-known technique that has been disclosed to the many commercial products currently being published, via multiple routes [5-8].

The camera used for the latest mobile phone secures image quality of a small DSLR level. Also in this cell phone camera, the above-mentioned certain correction function is added. However, when an ordinary person shoots using a mobile phone camera, in many cases, it takes pictures of shaking. For these reasons, the number of experts and general people using camera sliders is increasing. Conventional products have the inconvenience that the photographer moves directly in a manual form. In addition, the electric camera slider needs to install an additional button for automatic operation, and this button controls the operation of the slider. In this paper, in order to solve such problems, we improved the discomfort of button operation by using smartphone and camera slider, Bluetooth communication. It is a camera slider developed when tracking and photographing an object, which can be controlled by Bluetooth communication, but many operations have to be done, which will cause vibration of the stepper motor of the slider Become. For these reasons, we will shoot images that are being shaken. In this paper, we developed a camera slider with low cost and high performance, and the main functions are as follows.

First, it is control of the stepping motor through slider control using Bluetooth communication and adaptive PID control. As a result of photographing the object using the camera slider including such a function, it was possible to acquire a picture without tremor. The structure of this paper is as follows. It is described in order of slider production using Adoino uno board, PID control of stepping motor and conclusion of simulation.

\section{HARD WARE DESIGN}

The slide of the remote control camera which contains object tracking function consists of LM guide, control, servo motor and rail. 
Figure 1 shows a configuration diagram of a remote control slider, which can be classified into five.

The control unit used the Aduino Uno board which includes the Bluetooth communication function. The camera was fixed and used LM Guide for linear motion. The LM guide is a device that linearly transfers linear rails machined elaborately by one of machine parts, while balls in the block are rolling efficiently. This device is used in various fields such as 3D printer, semiconductor mounting, machining center and automatic door of subway. To combine the LM guide and the control unit, we created an archive of the control unit using a 3D printer. It was fixed on the camera fixing part LM guide, and this part was also directly made using a 3D printer. Here the drawing of parts was designed using Auto CAD software so that it can be used in 3D printing. The stepping motor was used for the camera fixed to the LM guide and the government's linear movement. Although the stepping motor can precisely control compared with the servo motor and DC motor, vibration will occur. In this thesis, we designed PID controller to solve these vibrations and solved the vibration part. In traditional software development the project moves more clearly from a requirements/specification phase, through successive designs that are evaluated and refined, until the system is built. In Web development, there is far less clarity in these phases, with significant overlap. Designs are part of the build process, and lead through evaluation to a modification of specifications. Designs become successively deeper, moving from flat screens to functional prototypes, and there is an unclear distinction between the design process and the specification process, as in figure 1.

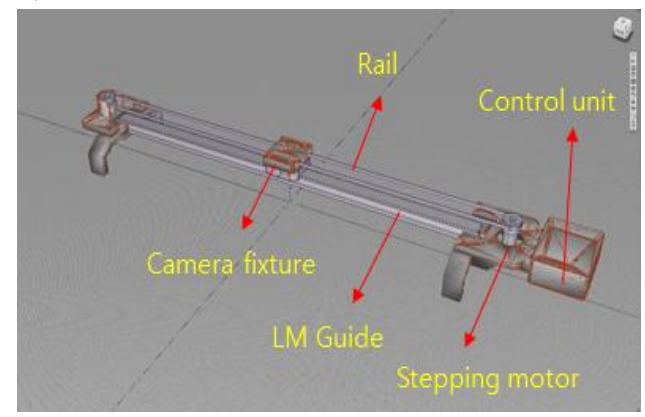

Fig 1 : Configuration diagram of remote control slide

\section{A. Remote Control Slide Behavior}

The operation of the remote control camera slider starts with pairing Aduino Uno board which is the control part and Bluetooth of the smart phone. Using the application of the next smartphone, send an action signal to the stepping motor with the action button Nulumyeong, Aduiono Uno board. At this time, before the signal is transmitted to the stepping motor, it is adjusted to the value of the PID controller to drive the stepping motor. Finally, the operation was automatically stopped at the end point of the camera slider and it was produced. Figure 2 shows a flowchart of the basic operation described above.

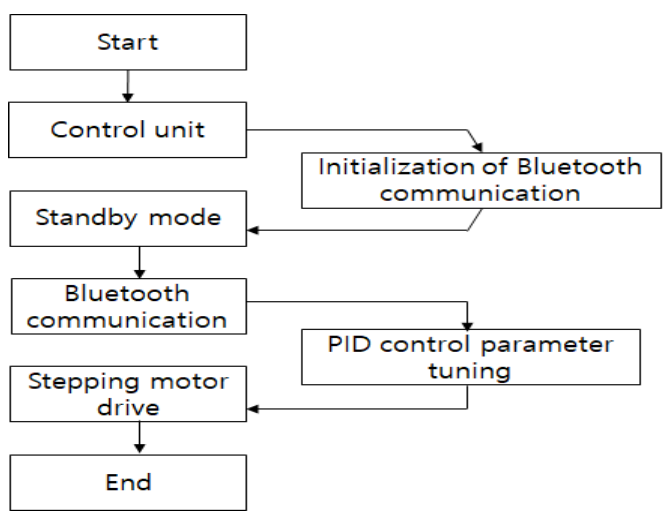

Fig 2 : Remote control slide operation flowchart

\section{B. Stepping Motor Control}

In the stepping motor control method, after steering the PID control parameter, an operation signal is applied to control the stepping motor. Here, instead of using the transfer function of the stepping motor, it is classified into a model reduction method using the least squares method, a response of the transient state of the output signal and a steady state response using the input / output data, We applied an algorithm to First order plus time delay (FOPTD) [9-12].

\section{Estimation of first order delay model using least squares method}

The least squares method (LSE) is well known as an algorithm for estimating a model. In this method, coefficients of the model are estimated so that the sum of squared errors is minimized using a set of input / output data of the process. The data commonly used here uses sampling data, and the model consists of difference equations. Since the structure used in this paper is a continuous time system, conversion between continuous time and discrete value system is required. In this paper, we propose a method to estimate continuous time FOPTD using LSE, which is the same as in figure 3 .

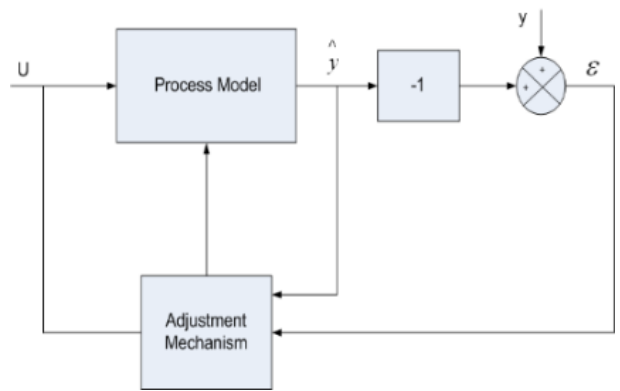

Fig 3 : Block diagram of LSE

The LSE obtains the coefficients of the difference equation, converts the difference equation to the discrete time state equation, converts the discrete time state equation to the continuous time state equation, converts it into the transfer function, FOPTD system can be obtained. The system that you want to obtain from the response of the process is the same as equation (1). 


$$
\frac{Y(s)}{U(s)}=\frac{k}{\pi s+1} e^{-s L}
$$

The delay time of Expression (1) can be written as Expression (2) using the Pade approximate expression.

$$
\frac{Y(s)}{U(s)}=\frac{-2 k s+k L}{2 \pi s^{2}+(\tau+2) s+L}
$$

First, the advantage value $\mathrm{k}$ of the process can be obtained as follows.

In the open loop adding a step input of size $\Delta \mathrm{m}$ to step $\mathrm{G}$ (s), the loop transfer function is the same as equation (3).

$$
c(s)=M(s) G(s)=\frac{k \Delta m e^{-\theta s}}{s(\tau s+1)}
$$

When $\mathrm{t} \rightarrow \infty$ is applied to equation (3), the process gain is the same as equation (4).

$$
k=\frac{\Delta c_{s}}{\Delta m}
$$

\section{Model reduction algorithm by classification of transient response and steady state response}

In order to obtain a single model including transient response and steady state response, to represent oscillating system. There are many errors. In this paper, in order to overcome the limits of these primary reduction models, a reduced model is obtained by classifying the response of the model as a transient response and a normal response, and the transient response part is further classified and reduced Proposal is as follows.

Step 1: Classification of response time of transient state of time response and response time of steady state

Looking at the response curve of the open-loop transfer function, the shape is as shown in figure 4 , so if you use such characteristics, mouth. The value of the output data can be classified into transient state response and steady state response, and the time to reach the steady state is the same as in equation (5).

$$
\lim _{t-x} y(t)=k
$$

Here, $x$ is the time at which the value of $y(t)$ first reached the value of process gain $\mathrm{k}$.

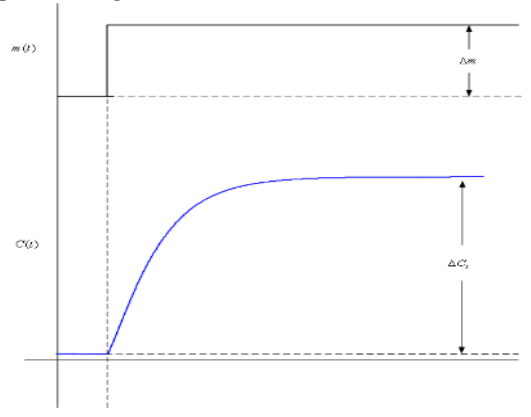

Fig 4 : Open-loop response curve
Here, the input is step response, and the advantage of the steady state is the value of $\mathrm{k}$ found in the previous chapter. That is, the time to reach the normal state means the time to first reach the normal state advantage $\mathrm{k}$ value.

Step 2: Reduced model of response part of transient state

It is a transient state response that accompanies the maximum error between a higher order model and a reduced model having a first order delay time. This is because the roots of the characteristic equation in the case of the higher order model include not only the actual route and the double root, but also Hogun. In order to solve the case where the higher order model includes the Hoggun of the characteristic equation, this paper proposes a method of classifying the response part of the transient state into certain sections and obtaining the reduced model suitable for each section. It is as shown in equation (6).

$$
y_{t}(t)=y_{t 1}(t)+y_{t 2}(t)+y_{t 3}(t)+\cdots+y_{t n}(t)
$$

Step 3: Reduced model of steady state response part

The steady-state response of the higher order model is the response after reaching the advantage of the process, so the gain of the process is maintained. Therefore, in this case, it can be sufficiently expressed by the reduced model of one FOPTD, which is the same as the expression (7).

$$
y_{s s}(t)=\hat{y}_{s s}(t)
$$

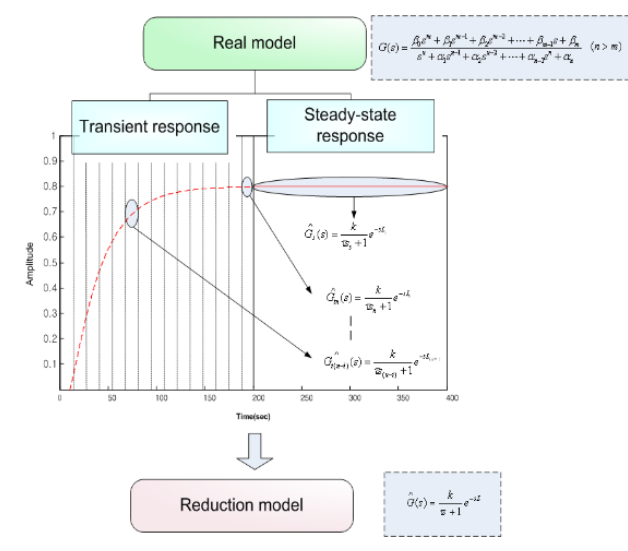

Fig 5 : Model Identification Algorithm

The algorithm of the proposed reduced model is the same as in figure 5 in this paper.

\section{RESULT AND DISCUSSION}

\section{A. Stepping motor modeling and PID parameter tuning}

In this paper, the parameter identification of the stepping motor is modeled in consideration of the transient response and the steady state response, and the PID parameter tuning is designed to optimize the ITAE value based on the analytical method.

The reduced model considering the transient response and steady state response by the unprocessed method is as follows. 


$$
\widehat{G}_{p 1}(s)=\frac{k 2}{\tau 2 s+1} e^{-s L 2}
$$

As for the controller parameter, a single reduced model was applied, and the value obtained by minimizing the figure of merit ITAE value and the optimized PID parameter proposed in this paper are shown in Table 1 and Table 2.

Table- I:The proposed model tuning

\begin{tabular}{|c|c|c|c|c|}
\hline \multirow{2}{*}{$\hat{G}_{p 1}(s)=\frac{k 2}{\tau 2 s+1} e^{-s t 2}$} & & \multicolumn{3}{|c|}{ Model Parameters } \\
\hline & & $k 2$ & $\tau 2$ & L2 \\
\hline \multirow{6}{*}{$\begin{array}{l}\text { Transient } \\
\text { state } \\
\text { Response }\end{array}$} & 1 & 1 & 6.8662 & 4.2498 \\
\hline & 2 & 1 & 5.5316 & 4.1838 \\
\hline & 3 & 1 & 6.1970 & 4.1178 \\
\hline & : & : & : & : \\
\hline & 11 & 1 & 3.5202 & 3.5898 \\
\hline & 12 & 1 & 3.1860 & 3.5240 \\
\hline $\begin{array}{c}\text { Steady state } \\
\text { Response }\end{array}$ & & 1 & 3.1856 & 3.5238 \\
\hline
\end{tabular}

Table- II: Controller parameter

\begin{tabular}{|c|c|c|c|c|}
\hline \multirow{2}{*}{$\tilde{G}_{\mathrm{eI}}(s)=v \frac{a s+b}{s}$} & & \multicolumn{3}{|c|}{ PID Parameters } \\
\hline & & $\boldsymbol{k}_{p}$ & $\boldsymbol{k}_{i}$ & $k_{\bar{L}}$ \\
\hline $\begin{array}{c}\text { Conventional } \\
\text { method }\end{array}$ & & 1.4481 & 9.7112 & 1.3534 \\
\hline \multirow{6}{*}{$\begin{array}{l}\text { Transient } \\
\text { state } \\
\text { Response }\end{array}$} & 1 & 1.4509 & 9.7348 & 1.3543 \\
\hline & 2 & 1.4092 & 9.3022 & 1.3300 \\
\hline & 3 & 1.3659 & 8.8699 & 1.3056 \\
\hline & : & $:$ & $:$ & $:$ \\
\hline & 11 & 0.9491 & 5.4441 & 1.1041 \\
\hline & 12 & 0.8860 & 5.0255 & 1.0780 \\
\hline $\begin{array}{c}\text { Steady state } \\
\text { Response }\end{array}$ & & 0.8857 & 5.0250 & 1.0776 \\
\hline
\end{tabular}

Figure 6 shows the open loop step response of the conventional model reduction method and the proposed model reduction method. In this case, it can be confirmed that the proposed method has less error between the transient state and the steady state than the conventional method.

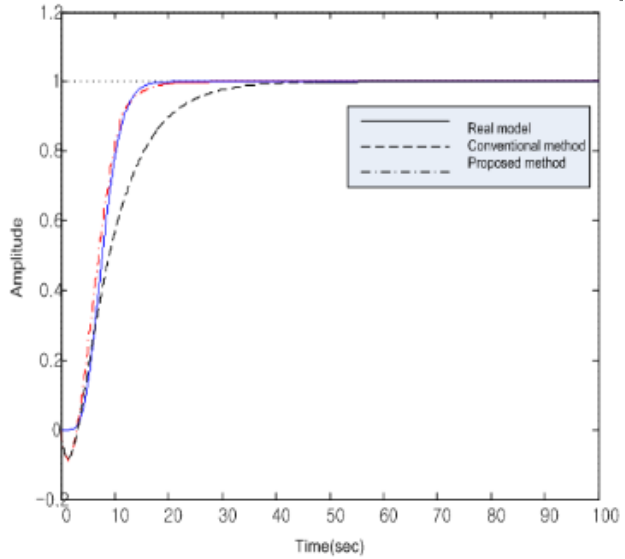

Fig. 6. Open- loop time response comparison

\section{B. Remote control slide behavior}

The specifications and figures of the completed remote control slide are as follows.

Standard: width $660 \mathrm{~mm}$, length $135 \mathrm{~mm}$, height $71 \mathrm{~mm}$, weight $2 \mathrm{~kg}$
Rail length: $500 \mathrm{~mm}$, width: $15 \mathrm{~mm}$, load: $10 \mathrm{KN}=1,020.4$ kgf

Auxiliary battery power supply, smart phone remote control possible (position and speed)

Figure7 shows a movie using the slide of the remote camera that we made, capturing a movie with no screen shake so that there is no shortage compared with existing products.

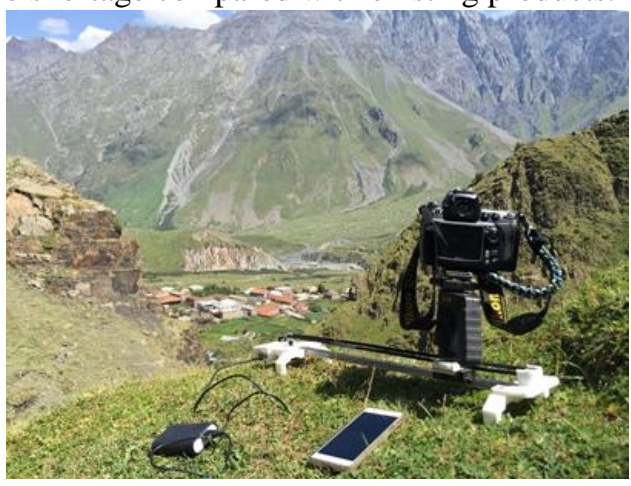

Fig. 7. Slide of remote camera that can communicate with Bluetooth

\section{CONCLUSION}

In this thesis, a slide of a remote camera was made. Technique made on the slide of the produced camera makes it possible to control the operation of the slide by using the Bluetooth of the smartphone, design the parameter tuning of the stepping motor and the adaptive PID controller, the vibration I made it work less. We showed that the slide of the remote camera applying such technology operates without vibrating when used at the actual site. In the future Zero must apply image processing with raspberry pie and OpenCV program to solve these problems.

\section{ACKNOWLEDGMENT}

In this paper was supported by Wonkwnag University in 2019.

\section{REFERENCES}

1. Y. Toyoda, Image stabilizer, U.S. Patent 6064827, 2000.

2. M. Yamamoto, Y. Yoko, and S. Haga, Image stabilization apparatus, U.S. Patent 7164531, 2007.

3. K. Washisu, Image stabilization device, U.S. Patent 5245378, 1993.

4. H. Hanselmann, " Implementation of digital controllers-A Survey", Automatica, vol. 1,no. 23, 1987, pp. 7-32.

5. Digital Control Applications with the TMS320 Family, Texas Instruments, 1991.

6. K. Ogata, Discrete-Time Control Systems 2/e, Prentice Hall, 1994. pp. 205.

7. K.J.Astrom and T.Hagglund, "Automatic tuning of simple regulators with specifications on phase and amplitude margins", Automatica, vol. 5, no.20, 1984, pp.645-651.

8. W.K.Ho, C.C.Hang, W.Wojsznis, and Q.H.Tao, "Frequency domain approach to self-tuning PID control", Contr.Eng. Practice, vol. 6, no. 4 1996, pp. 807-813.

9. W.K.Ho, O.P.Gan, E.B.Tay, and E.L.Ang, "Performance and gain and phase margins of well-known PID tuning formulas", IEEE Trans. Contr. Syst. Technol., vol. 4, 1996, pp. 473-477.

10. M.Zhuang and D.P.Atherton, "Automatic tuning of optimum PID controllers”, Proc. Inst. Elect. Eng., vo. 3, no.140, 1993,pp 216-224. 
11. K.J.Astrom, Automatic tuning of PID regulators, Instrument Soc. Amer., 1998,pp. 115

12. K.Y.Kong, S.C.Goh, C.Y.Ng, H.K.Loo, K.L.Ng, W.L. Cheong, and S.E.Ng, Feasibility report on frequency domain adaptive controller, Singapore, 1995. pp. 238

\section{AUTHORS PROFILE}

Author-1

Photo

Joon-Ho Cho 2007 present: Associate Professor, Dept. of Electronic Convergence Engineering, Wonkwang University <Interests>Electric, electronics, robot vision, medical image processing. 